ERRATUM

Koji Azegami · Takanori Tsukamoto $\cdot$ Takayuki Matsuura

Tatsuji Ohara • Yasuhiro Inoue • Akifumi Mizuno

Kouji Yoshida $\cdot$ Hideo Bessho $\cdot$ Shigeru Kimura

Masao Goto

\title{
Invasion and colonization of mature apple fruit by Erwinia amylovora tagged with bioluminescence genes
}

\section{J Gen Plant Pathol (2004) 70: 336-341}

An error appeared in the article cited above.

In the Introduction, on page 336, the sentence beginning on the 8th line from the bottom of the right column was incorrectly shown as:

Recently, Mizuno et al. (2002) and Tsukamoto et al. (in press) showed that E. amylovora placed on the cut surfaces of fruit stems (pedicels) of commercially purchased apples migrated at least $8-13 \mathrm{~mm}$ downward along the pedicels, and that the flesh (mesocarp) rotted although the pathogen was not detected with modified Miller-Schroth medium.

The correct sentence is the following:

Recently, our group (T. Tsukamoto, T. Matsuura, S. Kimura, and M. Goto) showed that E. amylovora placed on the cut surfaces of fruit stems (pedicels) of commercially purchased apples migrated at least $8-13 \mathrm{~mm}$ downward along the pedicels, and that the flesh (mesocarp) rotted although the pathogen was not detected with modified Miller-Schroth medium described by Mizuno et al. (2002).

The publisher sincerely apologizes for the error.

K. Azegami $(\bowtie) \cdot T$. Matsuura · Y. Inoue

National Agricultural Research Center, Tsukuba, Ibaraki 305-8666,

Japan

Tel. +81-29-838-8931; Fax +81-29-838-8929

e-mail:migazea@affrc.go.jp

T. Tsukamoto $\cdot$ T. Ohara $\cdot$ A. Mizuno $\cdot$ S. Kimura

Yokohama Plant Protection Station, Yokohama, Japan

K. Yoshida

National Institute of Fruit Tree Science, Tsukuba, Japan

H. Bessho

National Institute of Fruit Tree Science, Morioka, Japan

M. Goto

Shizuoka University, Shizuoka, Japan 